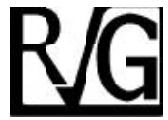

\title{
Dinamismo de las PYMES en Colombia: un estudio comparativo de empresas
}

\author{
Franco-Ángel, Mónica* \\ Urbano, David ${ }^{\star \star}$
}

\section{Resumen}

El propósito de esta investigación cualitativa es identificar los factores determinantes del dinamismo de ocho Pequeñas y Medianas Empresas (PYMES) colombianas, ubicadas en dos de las regiones más importantes del país, a través de un estudio de casos múltiples con una unidad de análisis. La información se obtuvo mediante entrevistas en profundidad con cada uno de los empresarios fundadores y con la segunda persona en la línea de jerarquía, así como de múltiples fuentes de evidencia y la observación. La información fue tabulada y categorizada mediante el software Atlas.ti. El marco conceptual de referencia incluye la teoría de recursos y capacidades, y la teoría institucional. Los resultados del estudio sugieren que los factores internos más relevantes son los recursos financieros, la reputación y el prestigio de las empresas frente a la comunidad, las habilidades específicas tanto del empresario como de los empleados y las capacidades organizativas estratégicas. Por otro lado, el factor del entorno que más incide en el dinamismo alcanzado por las empresas estudiadas es fundamentalmente la cultura relacionada con la influencia de la comunidad y de los familiares del fundador en el proceso empresarial. Se concluye que el dinamismo empresarial está en función de factores internos y externos. La diferenciación en los recursos y las capacidades organizativas estratégicas permiten la heterogeneidad de la PYME y la generación de ventajas competitivas.

Palabras clave: Dinamismo empresarial, PYMES, recursos, instituciones.

Recibido: 10-11-11. Aceptado: 12-11-13

* Doctora en Creación y Gestión de Empresas, Directora Programa de Administración de Empresas, Universidad Icesi, Cali (Colombia). E-mail: mfranco@icesi.edu.co. La autora reconoce el apoyo financiero de la Universidad Icesi para el desarrollo de la investigación.

** Doctor en Administración y Dirección de Empresas, Coordinador Postgrado en Creación de Empresas, Universidad Autónoma de Barcelona, Barcelona (España). E-mail: david.urbano@uab.es 


\title{
The Dynamism of SMEs in Colombia: A Comparative Study of Companies
}

\begin{abstract}
The purpose of this qualitative research is to identify determining factors for the dynamism of eight small and medium Colombian enterprises (SMEs) located in two of the most important regions of the country, through a study of multiple cases with one analysis unit. Information was obtained through in-depth interviews with each of the founding businessmen and with the person next-in-line in the hierarchy, as well as with multiple sources of evidence and observations. Information was tabulated and categorized using Atlas.ti software. The conceptual reference framework included the theory of resources and capacities, and institutional theory. Results of the study suggest that the most relevant internal factors are financial resources, reputation and the prestige of the companies in the eyes of the communities, specific abilities of both entrepreneur and employees and strategic organizational capacities. On the other hand, the factor in the surroundings that most affects the dynamism achieved by the studied enterprises is fundamentally the culture related to the influence of the community and family members of the founder on the business process. Conclusions are that entrepreneurial dynamism is related to internal and external factors. Differentiation in the resources and strategic organizational capacities permit the heterogeneity of the SMEs and the generation of competitive advantages.
\end{abstract}

Keywords: Entrepreneurial dynamism, SMEs, resources, institutions.

\section{Introducción}

Ibielski (1997) se refiere a las PYMES como sistemas poderosos reflejo del espíritu competitivo que una economía de mercado necesita para ser eficiente. Estas empresas se constituyen en una salida para el talento empresarial, poseen mayor flexibilidad y son más rápidas para reaccionar (Dhawan, 2001; Mitchell y Singh, 1993; Nooteboom, 1994). Sin embargo, la existencia de debilidades de carácter estructural disminuye su competitividad y condiciona su capacidad de supervivencia y crecimiento, presentándose en ellas elevadas tasas de mortalidad.
Por su parte, Franco y Haase (2009) indican que el fracaso de las PYME es alto en sus primeros años de vida, European Federation of Accountants- FEE (2004) aduce que las PYMES no mayores a tres años tienen un alto porcentaje de fracaso $y$ Baldwin et al (2000) plantean que el $23 \%$ de las empresas canadienses fracasan durante su primer año de existencia y solo un $42 \%$ tiene probabilidades de sobrevivir después de su cuarto año de operación.

En el caso particular de Colombia, contexto de la presente investigación, las estadísticas proporcionadas por el estudio Global Entrepreneurship Monitor (GEM, 2010) muestran que este país cuenta con 
una tasa de actividad empresarial del $20,6 \%{ }^{1}$. En este estudio, Colombia ocupa el tercer lugar en actividad empresarial más alta, entre los países estudiados a nivel mundial. Sin embargo, son muchas las empresas que desaparecen; según el estudio mencionado la tasa de mortalidad empresarial asciende a $10,5 \%$.

Es importante mencionar que en Colombia la distribución del tejido empresarial se encuentra conformada por microempresas (96,1\%), seguido de las pequeñas y medianas empresas $(3,8 \%)$ y las grandes empresas $(0,1 \%)^{2}$, y generan el 80,9\% del empleo, según datos del Departamento Administrativo Nacional de Estadística de Colombia-DANE (2005) $)^{3}$. A pesar de su importancia, en la mayoría de los casos muchas se encuentran en sus etapas iniciales de creación o quedan rezagadas en su crecimiento, con lo cual su contribución al desarrollo económico del país no es la esperada. En este sentido, las empresas que crecen y logran aumentar su competitividad son llamadas gacelas, de rápido crecimiento o dinámicas (Fischer y Reuber, 2003; St-Jean et al, 2008; Kantis, 2004), aunque realmente no existe una definición universalmente aceptada.
Así, Kantis (2004) define como dinámica a aquella empresa que en sus primeros 48 meses de vida ha incrementado su planta de personal en más de 15 empleados; St-Jean et al (2008) clasifican como gacelas a las empresas que presentan un crecimiento anual promedio del $20 \%$ en ventas al menos por cuatro años, y Fischer y Reuber (2003) definen como dinámicas a aquellas empresas con una tasa de crecimiento en ventas de al menos $20 \%$ por año, durante cinco años consecutivos.

Teniendo presente lo anterior, en este trabajo se denomina "empresa dinámica" a empresas que han tenido un crecimiento anual promedio en ventas del $20 \%$ en los últimos cinco años.

En este contexto, el propósito principal de la investigación es identificar los factores determinantes del dinamismo de las PYMES en Colombia. Se distinguen los factores internos (propios de la empresa) de los externos (o del entorno) a la luz de la teoría de recursos y capacidades y de la teoría institucional. Se analizan las regiones colombianas de Cundinamarca y del Valle del Cauca, las cuales concentran el $29,1 \%$ y el $9,6 \%$ respectivamente

1 La Tasa de Actividad Empresarial (TEA), mide el porcentaje de personas entre los 18 y los 64 años de edad activamente involucrados en el proceso de creación de una actividad empresarial.

2 La denominación de empresa Micro, Pequeña, Mediana y Grande en Colombia (MIPYME), viene determinada por la ley 590 de 2000 (Congreso de la República de Colombia, 2000) modificada por la ley 0905 de 2004, la cual determina: Empresa micro: planta de personal no superior a 10 trabajadores y activos totales por valor inferior a 501 Salarios Mínimos Mensuales Legales Vigentes (SMMLV). Empresa pequeña: planta de personal entre 11 y 50 trabajadores y activos totales por valor de entre 501 y 5.000 SMMLV. Empresa mediana: planta de personal entre 51 y 200 trabajadores y activos totales por valor de entre 5.001 y 30.000 SMMLV. Empresa grande: más de 200 empleados y activos mayores a 30.001 SMMLV.

3 A la fecha el DANE no ha actualizado las cifras sobre la distribución del tejido empresarial. 
de las empresas del país, lo que corresponde al 38,7\% del total de la población empresarial (DANE, 2005).

En cuanto a la metodología se lleva a cabo una investigación con enfoque cualitativo, realizando un estudio de casos de ocho PYMES colombianas. Para el análisis de los resultados, se realiza la transcripción de todas las entrevistas y cada pregunta se tabula y categoriza mediante el software Atlas.ti ${ }^{4}$, el cual permite el análisis cualitativo y la interpretación de grandes cuerpos de datos textuales, de gráficos y de video, facilitando su análisis visual.

\section{Teoría de recursos y capacidades y teoría institucional}

La teoría de recursos y capacidades y la teoría institucional se constituyen en el marco de referencia de este estudio. Se cuenta con la propuesta de Sirmon et al (2007), quienes aducen que la teoría de recursos y capacidades reside en la heterogeneidad o disparidad existente entre las empresas, surgida de la diferencia en sus recursos, capacidades o competencias básicas, y en su posible valor como fuente de ventaja competitiva sostenible. Sin embargo, los recursos y capacidades que desarrolla o posee una empresa no pueden considerarse en su totalidad como estratégicos. Morgan et al (2004), King (2007) y Ainuddin et al (2007), sostienen que no todos los recursos que poseen y controlan las empresas tienen el potencial para conducir a un desempeño superior, y que aquellos generadores de beneficios extraordinarios contribuirán con el logro de la ventaja competitiva de las empresas.

Por otra parte, estos recursos estratégicos intervienen en la creación de capacidades que permiten la obtención de ventajas competitivas. Dichas capacidades son rutinas organizativas como resultado de la transferencia de conocimiento al interior de las empresas. Autores tales como King (2007), Perez-Freije y Enkel (2007) y Sirmon et al (2007), muestran en sus estudios la relación entre las capacidades que poseen las empresas y su ventaja competitiva.

Estudios empíricos de autores como Al-Mahrouq (2010), Carmeli y Tishler (2004), Franco-Ángel y Urbano (2010), Min et al (2005) y, Piva y Vivarelli (2002), entre otros, confirman la importancia que representan para el dinamismo de las empresas los recursos tangibles, intangibles y humanos, y las capacidades organizativas.

En forma complementaria para este estudio, la teoría institucional destaca la importancia económica de los hábitos de conducta y del pensamiento de los grupos humanos, y analiza el tema de las instituciones sociales (North, 1990; Meyer et al, 2009; Welter y Smallbone, 2011). De acuerdo con el enfoque de North $(1990,2005)$ el concepto de "instituciones" se desarrolla para referirse a los factores externos, y se constituyen en normas y reglas restrictivas que rigen la 
sociedad, condicionando y dirigiendo el marco de las relaciones producidas en ella. Para dicho autor, dado que la información disponible es incompleta y la capacidad mental del individuo para procesar, organizar y utilizar la información es limitada, las instituciones concebidas por el ser humano para estructurar sus relaciones, limitan el conjunto de elecciones que ofrecen a los actores reduciendo la incertidumbre.

De esta manera, las instituciones forman la estructura de incentivos de una sociedad y, por tanto, son estas las que determinan el desempeño en el largo plazo. Así, las instituciones formales incluyen normas políticas, legales y económicas, y las instituciones informales están representadas por ideas, creencias, actitudes y valores de las personas, las cuales constituyen la cultura de una determinada sociedad.

Por otra parte, varios autores confirman la relevancia de los factores del entorno como condicionantes de la creación de empresas desde el enfoque institucional (Aidis et al, 2008; Alvarez y Urbano, 2011; Alvarez et al, 2011; Thornton et al, 2011; Welter y Smallbone, 2011), especialmente en el dinamismo empresarial (Ahn y York, 2011; Meyer et al, 2009; Tödtling et al, 2011).

Partiendo de la revisión de literatura, las variables a estudiar en la presente investigación son definidas en función de los recursos y capacidades estratégicas así como de las instituciones formales e informales, presentando en los Cuadros 1 y 2 la relación de dichas variables.

\section{El dinamismo de las PYMES en Colombia}

Se presenta ahora el análisis de la información obtenida inicialmente en forma individual, es decir, para cada caso particular, y después en forma cruzada para el grupo de casos realizados, para llegar a la discusión de los resultados y a la generalización de las conclusiones. Las empresas se identifican con las letras A, B, $C, D, E, F, G$ y $H$ para guardar la confidencialidad solicitada por los empresarios.

Tal como se menciona en la introducción, el análisis de la información se realiza con la ayuda del software Atlas.ti, el cual permite establecer la relación entre las diferentes variables estudiadas. Una vez que la información es tabulada y categorizada en dicho software, se codifican los párrafos alusivos a cada categoría de análisis, para obtener relaciones múltiples en la información de las oraciones y en el cruce con las categorías, consistentes con el marco teórico.

De acuerdo a las entrevistas realizadas a los fundadores y al personal directivo de las empresas, según los datos cuantitativos presentados por estas, y conforme al parámetro establecido en esta investigación para determinar el dinamismo empresarial (crecimiento anual promedio en ventas del $20 \%$ en los últimos cinco años), se define la clasificación de cada empresa como dinámica o no dinámica (Tabla 1):

El Cuadro 3 presenta los factores internos y externos que según la percep- 


\section{Cuadro 1}

\section{Factores Internos: Recursos y Capacidades Estratégicas}

\begin{tabular}{lll}
\hline Variables Indicador \\
\hline \multicolumn{2}{c}{ Recursos Tangibles } \\
\hline
\end{tabular}

Activos físicos

Tamaño de la empresa

Número de empleados y monto de activos totales

Localización

Ubicación de la sede, propiedad

Equipo y maquinaria

Tipologías de equipos y maquinaria, sofisticación técnica, flexibilidad de la planta

Activos financieros

Indicadores financieros

Capital

\section{Recursos Intangibles}

Reputación y prestigio de la empresa Activos intangibles

Posicionamiento de la empresa frente a la comunidad

Cultura organizacional Impacto de los valores, creencias, comportamientos de la empresa y sus empleados

$\underline{\text { Derechos de Propiedad }}$

Patentes y/o marcas que posee y/o utiliza

\section{Recurso humano}

Empresario y su equipo fundador Edad

Calificaciones del empresario y su equipo fundador

Características y habilidades del empresario y su equipo fundador

Experiencia

Presencia de padres empresarios

Presencia de familiares empresarios

Presencia de socios

Equipo de trabajo

Calificaciones y, destrezas y habilidades de los empleados.

\section{Capacidades Organizativas}

Áreas funcionales de la empresa

Actividades

Recursos

Capacidades organizativas
Actividades en cada una de las áreas funcionales

Recursos utilizados en cada área funcional

Capacidades organizativas desarrolladas en cada área funcional

Fuente: Elaboración propia. 


\section{Cuadro 2}

Factores Externos: instituciones formales e informales

\begin{tabular}{ll}
\hline Variables & Indicador \\
\hline \multicolumn{2}{l}{ Instituciones Formales } \\
\hline
\end{tabular}

Política económica y social

Medidas de apoyo

Legislación sobre desarrollo empresarial

Características del sector industrial

Tamaño y concentración del mercado

Competencia

Tecnología

Especialización
Conocimiento sobre política económica y social

Impacto de la política económica y social en el desempeño de la empresa

Beneficios que ha recibido la empresa por la política económica y social

Conocimiento sobre medidas y entidades de apoyo, y su utilización

Conocimiento sobre la legislación empresarial

Beneficios e impacto de la legislación empresarial en el desempeño de la empresa

Cantidad y tipologías de empresas

Competidores directos e indirectos

Formas de negociación con clientes y proveedores

Participación de la empresa en el mercado

Tecnología utilizadas

Grado de diferenciación del producto de la empresa

\section{Instituciones Informales}

\section{Cultura}

Influencia de la comunidad

Visión de la comunidad frente a la importancia del empresario y de las PYMES en la sociedad

Impacto de la comunidad en la empresa y de esta en la comunidad

Influencia de familiares y amigos

Apoyo de familiares y amigos en el proceso empresarial

en la creación y en el desempeño

de la empresa

Influencia de la cultura regional

Impacto de los valores, creencias y comportamientos culturales

Participación en redes sociales y em- Impacto de la participación en redes empresariales y presariales sociales

Fuente: Elaboración propia. 


\section{Tabla 1}

\section{Clasificación de las empresas de la muestra según el indicador de dinamismo}

\begin{tabular}{ccccc}
\hline Empresa & Ciudad & $\begin{array}{c}\text { Crecimiento en } \\
\text { ventas (\%) }\end{array}$ & $\begin{array}{c}\text { Crecimiento en } \\
\text { número de } \\
\text { empleados }\end{array}$ & Clasificación \\
\hline A & Cali & 629,0 & 196 & Dinámica \\
B & Cali & 114,0 & 30 & Dinámica \\
C & Cali & 18,4 & 9 & No Dinámica \\
D & Cali & 15,0 & 9 & No Dinámica \\
E & Bogotá & 38,8 & 37 & Dinámica \\
F & Bogotá & 33,4 & 26 & Dinámica \\
G & Bogotá & 16,0 & 15 & No Dinámica \\
H & Bogotá & 8,0 & 18 & No Dinámica \\
\hline
\end{tabular}

Fuente: Elaboración propia.

ción de los empresarios y ejecutivos influyen de manera decisiva en el dinamismo de las empresas. Como puede observarse, las empresas estudiadas cuentan con más de 42 meses de existencia, y pertenecen a diferentes sectores económicos; cuatro de ellas se encuentran ubicadas en Cundinamarca y otras cuatro en el Valle del Cauca. En cada una de estas regiones, se cuenta con dos empresas dinámicas y dos no dinámicas. Los resultados muestran que los fundadores consideran la importancia de los factores internos y externos en el dinamismo de sus empresas, y aunque algunos de éstos son seleccionados por todos ellos, su presencia resulta más significativa en las empresas consideradas como dinámicas.

Para el análisis cruzado de los casos, a continuación se discute el impacto en las empresas de cada una de las variables consideradas. Así, con relación a los factores internos, que como se mencionó anteriormente, están definidos en función de los recursos y capacidades estratégicos, se analizan las siguientes variables, haciendo énfasis en aquellas que han impactado mayormente en el dinamismo de las empresas estudiadas:

Activos físicos: todas las empresas estudiadas funcionan en sedes que en tamaño y ubicación resultan apropiadas para su operación, sólo tres de ellas, tienen propiedad sobre la misma. En términos generales, el espacio físico más grande se destina para el funcionamiento del área de producción; las oficinas administrativas son pequeñas e informarles; y la maquinaria y equipo son especializados pero no de alta tecnología, lo que coincide con el estudio GEM (2010) y confirma que en Colombia el porcentaje de empresas con tecnologías de punta aplicada en sus procesos de producción es inferior al $20 \%$.

Así mismo, la mayoría de las empresas cuenta en su proceso operativo con un componente manual significativo, por lo cual, aunque consideran la maquinaria importante, le dan mayor valor a la 


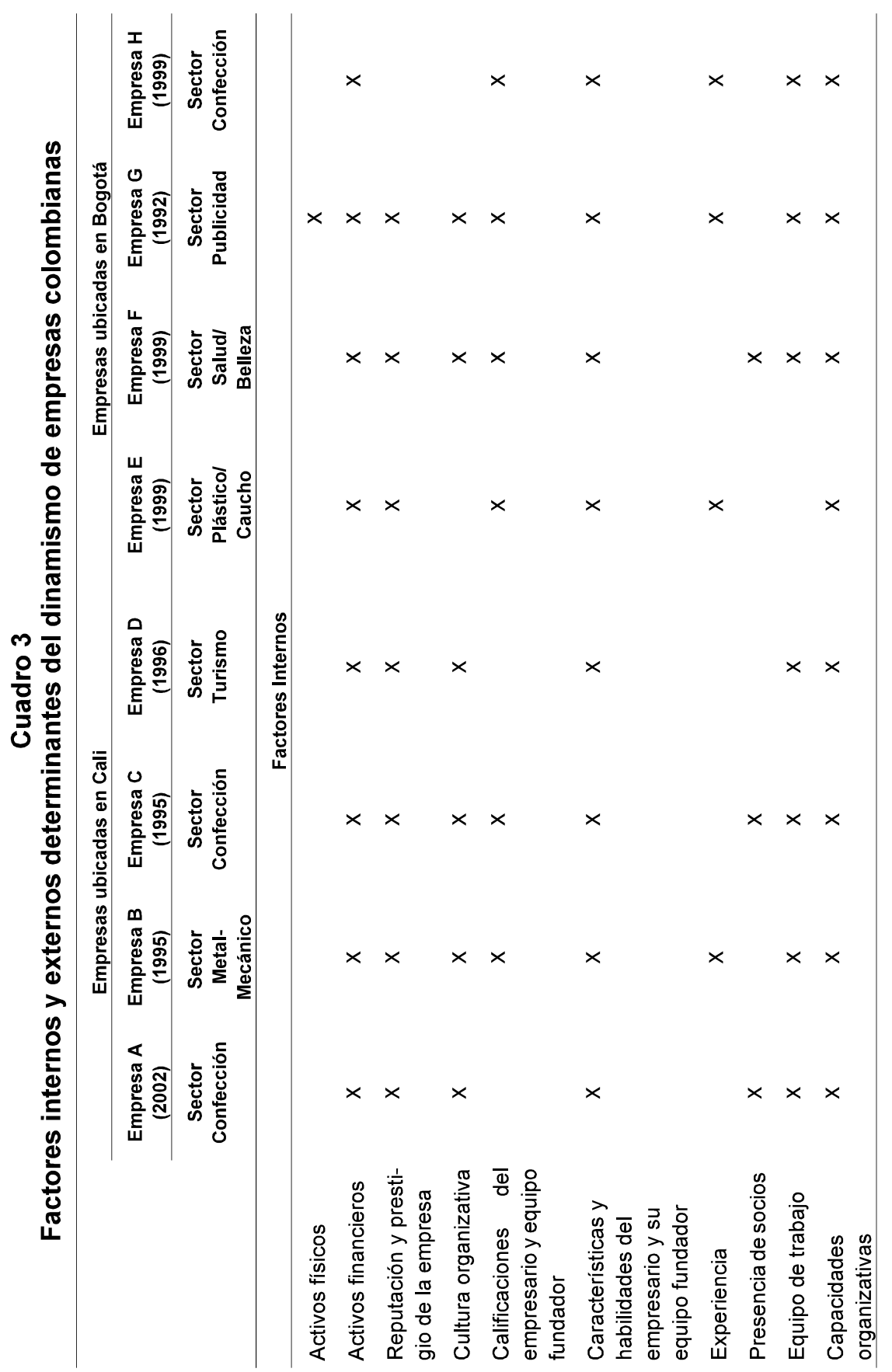




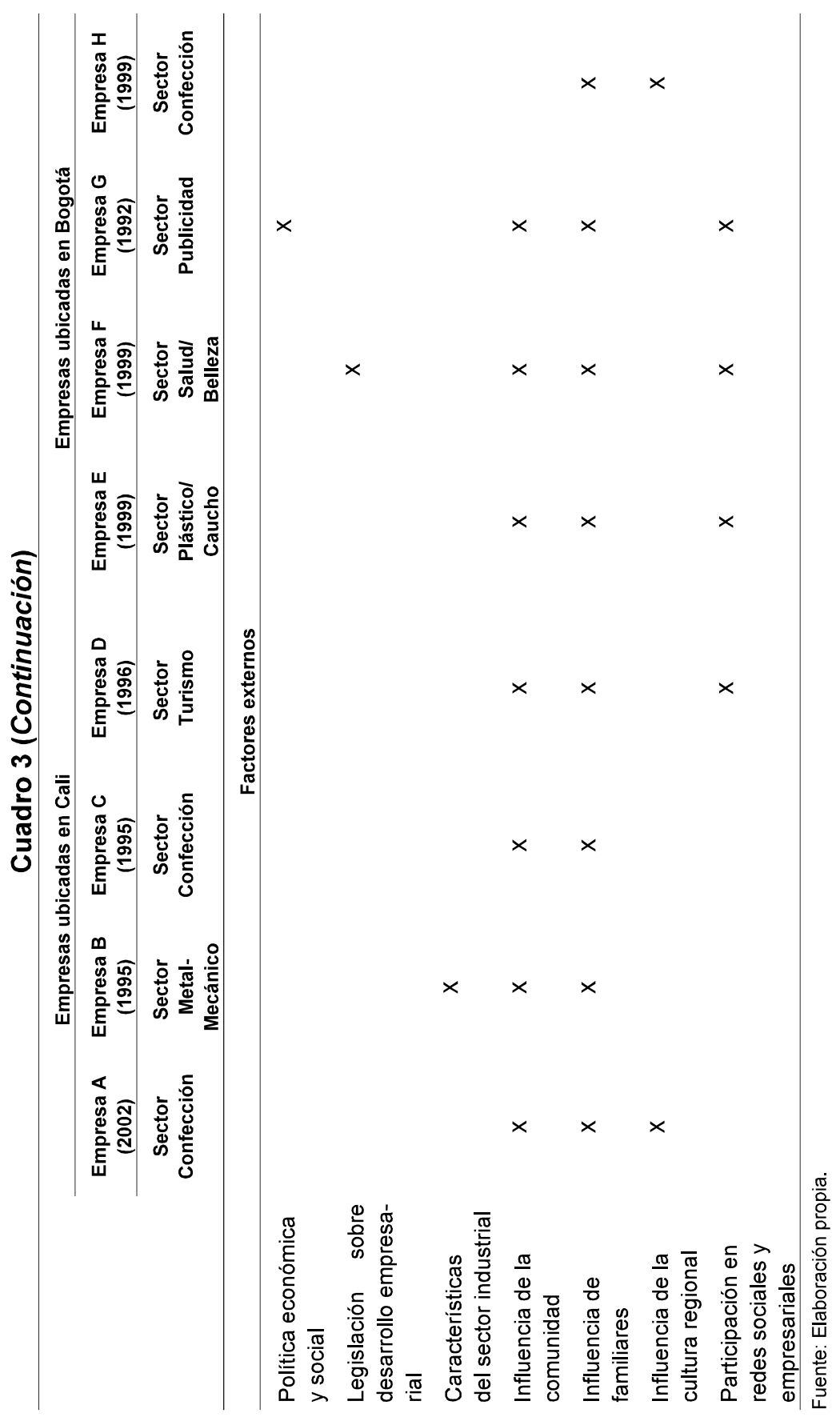


especialización de la mano de obra. Algunas de las empresas utilizan talleres externos para maquilar parte de sus procesos, aprovechando la infraestructura y la tecnología de estos. La estructura física encontrada en estas empresas es común para las PYMES colombianas dada su limitación en recursos de capital, razón por la cual los empresarios prefieren invertir el dinero en compra de materia prima, pago de salarios y adquisición de inventarios.

Activos financieros: los empresarios coinciden en la importancia de contar con el capital de trabajo para funcionar, y realizar las inversiones exigidas por el crecimiento, lo cual confirma con los estudios de Morgan et al (2004) y Ainuddin et al (2007) quienes evidencian que los recursos financieros tales como el flujo de caja positivo, los depósitos y ahorros en bancos y el capital financiero como las acciones, explican el nivel de ventaja competitiva y desempeño de las empresas. Para Min et al (2005) el factor más representativo en el fracaso de una empresa es la carencia de capital.

En las empresas menos dinámicas puede notarse que la carencia de una estructura financiera genera iliquidez, pagos atrasados a proveedores y al Estado, e incumplimientos con los bancos. En contraste, las empresas dinámicas presentan mayor organización en el área financiera $y$, especialmente, características gerenciales que les permite manejar este recurso eficientemente. El indicador preferido por los empresarios para conocer el estado de sus empresas es el índice de ventas, lo que resulta apenas lógico pues los ingresos están limitados al nivel de ventas que tengan. Es usual la práctica de reinvertir las utilidades para la capitalización, especialmente en las primeras etapas de la empresa, hallazgo que se considera relevante pues en general los pequeños empresarios tienden a descapitalizar la empresa al no hacer diferencias entre las finanzas personales y las de la empresa (Franco-Ángel y Urbano, 2010).

Los empresarios califican esta variable como muy importante. No obstante, Franco-Ángel y Urbano (2010), en un estudio previo sobre PYMES dinámicas del sector salud, encuentran que esta variable es considerada por los empresarios como poco relevante para el dinamismo de las empresas, pues para ellos es más importante la habilidad para adquirir y administrar estos recursos.

La reputación y el prestigio de las empresas frente a la comunidad, especialmente con clientes y proveedores, genera legitimidad y permite su posicionamiento en los mercados. Todos los empresarios están de acuerdo en afirmar que la etapa inicial de sus empresas fue difícil, pues no eran conocidos en la industria, y por tanto, no podían acceder fácilmente a los créditos de bancos y proveedores. Con el tiempo, los empresarios y las empresas adquieren aprendizaje y experiencia, y un mayor reconocimiento ante la comunidad. Sin embargo, en las empresas con mayor dinamismo se nota mayor reputación y prestigio $\mathrm{y}$, por ende, mayor posicionamiento, hallazgo que se confirma con los estudios de Carmeli y Tishler (2004) en el que la reputación organizativa aparece como un elemento crítico para que la organización alcance sus objetivos y logre un desempeño superior $\mathrm{y}$, Welter y Smallbone (2011) quienes sugieren que el contexto social influye en el comportamiento de los empresarios. 
Con relación a las empresas no dinámicas, se observa que aunque tienen clientes fieles y proveedores de muchos años, existe resistencia por parte de los empresarios a implementar estrategias agresivas de penetración de mercados, de inversión, y de ampliación de su base de clientes y proveedores. Los empresarios consideran esta variable como muy importante. Es relevante que los empresarios consideren la reputación y el prestigio de sus empresas como un factor determinante del dinamismo, pues la comunidad, entendida como clientes, proveedores y actores involucrados con la empresa, tiene poder para determinar las posibilidades de supervivencia y crecimiento de la misma.

Influencia de la cultura organizativa: para la mayoría de los fundadores el clima organizativo impacta en el desempeño de sus empresas, tal como lo demuestra en su estudio Hofstede (1994). Se puede ver que las relaciones entre propietarios y empleados son cálidas y se basan en la confianza y en la buena voluntad. Esta variable es calificada por ellos como muy importante.

Derechos de Propiedad: las empresas estudiadas no cuentan con patentes para sus productos pues argumentan que al ser éstos de fácil imitación no resultaría una buena inversión. En general, las empresas otorgan poca importancia a esta variable, aunque las marcas están registradas a nivel nacional. Este resultado no sorprende; Colombia es un país que presenta bajos índices de competitividad respecto a variables como derechos de propiedad y protección de la propiedad intelectual, tal como lo presenta Schwab (2011). En el caso de las PYMES, espe- cialmente en aquellas más nuevas y de menor tamaño, éste es un tema que no presenta prioridad y es considerado más como un gasto que como una inversión. Adicionalmente, dada la baja innovación de sus productos en muchos de los casos las patentes no tienen cabida.

El empresario y su equipo fundador: en el grupo de empresarios se nota un alto nivel educativo, todos cuentan con experiencia previa, pero sólo cinco de ellos en la industria a la que pertenece su empresa. Es evidente, aunque los fundadores de las empresas dinámicas no lo definen como muy importante, que esta experiencia les permite tener mayores herramientas para la gestión.

En cuanto a las características y habilidades del equipo fundador al crear la empresa y en la actualidad, los propietarios de las empresas dinámicas las consideran como un recurso valioso que les permite la dirección de las mismas hacia el crecimiento, y aducen que éstas cambian con el paso del tiempo, dados el aprendizaje y la experiencia. Los fundadores de las empresas dinámicas presentan mayor nivel de confianza en ellos mismos, más amplia visión de futuro y fuerte orientación al mercado. En este sentido, Wiklund et al (2009) sostienen que las actitudes de los empresarios y su orientación empresarial, se encuentran entre los factores con mayor influencia en el crecimiento de las PYMES.

En cuanto a la implementación de la estrategia, no se observa en las empresas mayor planeación, pero sí que el direccionamiento de las mismas obedece al pensamiento estratégico del empresario y a sus características y habilidades personales. En general, se evidencia que 
las estrategias perseguidas por los fundadores de las empresas dinámicas son la concentración en nichos de mercado y la diferenciación, coincidiendo de este modo con los estudios de Oyedijo (2012) y Suliyanto et al (2010). Dichas estrategias son concebidas por los fundadores de las PYMES pues son conscientes que para mantenerse en los mercados deben especializarse y atender clientes específicos lo cual les permite diferenciarse frente a empresas más grandes. FrancoÁngel y Urbano (2010) confirman que las estrategias de especialización y concentración en nichos de mercado, permite a las PYMES mayor competitividad.

Equipo de trabajo: los empresarios en su mayoría, reconocen que el apoyo de sus empleados, su especialización y su sentido de pertenencia contribuyen al logro de la ventaja competitiva de la empresa, resultado similar al evidenciado por Al-Mahrouq (2010) y Franco-Ángel y Urbano (2010). Cabe mencionar, que entre las empresas tanto dinámicas como no dinámicas se encuentran diferencias en cuanto al estilo de dirección de los empresarios. Los fundadores de las empresas dinámicas son de estilos paternalista, autoritario y participativo, y los de las no dinámicas, paternalista y participativo. Aunque los empresarios reconocen en su equipo de trabajo un recurso valioso, se observa en el interior de las empresas que el clima organizativo es diferente lo cual puede relacionarse con el estilo de dirección.

En cuanto a la transferencia de conocimiento, en todas las empresas existe marcadamente en el área de producción u operativa; esta situación es usual en las PYMES en las que, dada su orientación a los procesos y no a los mercados, el área operativa es la que mayor trascendencia tiene. Se comprueba así mismo la profesionalización, pues en las empresas en el área de gestión y dirección hay profesionales; sin embargo, dicho perfil corresponde a los propietarios y a sus familiares, pues en general, existe una muy baja proporción de empleados con educación superior.

En Colombia la profesionalización de las PYMES se ha incrementado dado que cada vez son más los profesionales que crean sus empresas. Es evidente de igual manera, que la retención de empleados se vuelve crítica para estas empresas dadas la falta de mano de obra especializada en las diferentes industrias y la carencia de recursos suficientes para contratar personal con mejores sueldos, lo cual se convierte en una gran debilidad para las mismas, pues tal como lo confirman Zelealem y Jaloni (2005), la consecución y el desarrollo del recurso humano constituye un factor crítico para el dinamismo de las PYMES. Esta situación se presenta no sólo en estas empresas, sino en general en todas las PYMES del país, en las cuales los niveles salariales son muy bajos con respecto al mercado; en primer lugar por la escasez de recursos y en segundo lugar porque muchos empresarios prefieren sacrificar el potencial de sus empresas contratando personas con bajo nivel académico, antes que disminuir sus utilidades por la erogación de sueldos.

Capacidades organizativas: los empresarios en su mayoría, consideran como las más importantes, las desarrolladas en las áreas de producción y comercial. En las empresas dinámicas se nota mayor 
desarrollo de las áreas de administración y finanzas. Es cierto, además, que las empresas dinámicas logran diferenciarse de otras empresas del sector, dada la forma como enfrentan la gestión de cada área, tal como lo reportan Piva y Vivarelli (2002), y por el desarrollo de recursos y capacidades idiosincrásicos, como lo discute la teoría de recursos y capacidades.

También se observa que el buen desempeño de las áreas depende de las características personales de quienes las dirigen que en su mayoría son los fundadores. Aunque las empresas evolucionan desde su nacimiento, pues tienen mayor formalización, procesos más organizados y un número más grande de empleados, los empresarios aún se encargan de la mayoría de las actividades de la gestión. En todas las empresas estudiadas se evidencia una orientación mayor hacia los procesos que hacia los mercados, situación presente comúnmente en las PYMES pues los nuevos empresarios, en su mayoría, crean sus empresas partiendo de sus conocimientos técnicos, por tanto, el mayor esfuerzo se dirige al desarrollo de los productos.

Respecto al análisis de los factores externos, definidos en función de las instituciones formales e informales, tal como ya se mencionó, se analizan las siguientes variables, enfatizando en aquellas que son consideradas por los empresarios como las de mayor impacto en el dinamismo de sus empresas:

Política económica y social: la mayoría de los empresarios considera que la política macroeconómica presenta un impacto negativo pues la carga impositiva es muy alta, y algunos de ellos, manifiestan que el sector PYME en Colombia se encuentra desfavorecido en cuanto a las políticas que contribuyen a su desarrollo. Esta percepción es generalizada entre las PYMES, a pesar del incremento de las políticas de apoyo para este sector, por parte del gobierno colombiano. Al respecto, Ahn y York (2011) evidencian la necesidad de mayor soporte por parte del gobierno para las PYMES, en relación con control de la inflación, disminución de impuestos e incentivos financieros, entre otros, y al respecto, Rodríguez (2003) encuentra que los trámites, la burocracia y los deficientes mecanismos de apoyo del Estado, son algunos de los grandes obstáculos que enfrentan las PYMES. En las empresas no dinámicas se observa un mayor desconocimiento de las políticas económicas y sociales.

Medidas y entidades de apoyo: aunque hay desconocimiento sobre las medidas, todos los empresarios utilizan los créditos bancarios, y la mayoría de ellos, conocen y se benefician de los programas de exportaciones del gobierno. Las entidades a las que más se recurre son los bancos y las Cámaras de Comercio. Se observa en las empresas una muy baja participación de asesores externos.

Legislación sobre desarrollo empresarial: los empresarios muestran gran desconocimiento de este tema y se observa desinterés por el mismo. En general, ellos consideran que las leyes en Colombia favorecen a las grandes empresas, y además, sienten gran desconfianza frente las entidades gubernamentales. Lo anterior, a pesar de que el gobierno colombiano ha incrementado los mecanismos de apoyo para este sector empresarial en la última década.

Características del sector industrial: aunque las empresas pertenecen a 
sectores económicos distintos, los empresarios coinciden en afirmar que la industria presenta alta concentración, requiere de especialización de la mano de obra y del producto final, y que el poder de negociación de su empresa, especialmente con los proveedores y con algunos de los clientes de mayor tamaño, es bajo. Además, aducen que el sector económico puede ejercer un impacto negativo, especialmente cuando la empresa es muy pequeña. Esta percepción de los empresarios encuentra soporte en el estudio de Kunc y Morecroft (2010), en el que se generan evidencias empíricas sobre la influencia de la estructura de la industria en el dinamismo empresarial.

Influencia de la comunidad: como ya se mencionó, los empresarios, en su mayoría, afirman que los proveedores y los clientes son los miembros de la comunidad con mayor impacto en el dinamismo de sus empresas, y reconocen que desarrollan una buena imagen frente a ellos. Este resultado es similar al obtenido en el estudio de Alvarez et al (2011) respecto a la importancia de la imagen social del empresario para la actividad empresarial. Se observa que los fundadores de las empresas dinámicas poseen mejores relaciones con sus clientes y proveedores lo cual les permite conocer las tendencias de los mercados y el comportamiento de la industria. En este sentido, Kunc y Morecroft (2010) confirman la importancia de la industria y del conocimiento de la competencia en el desempeño de las empresas y Gans et al (2008) reconocen que la estrategia enfocada en el cliente es creadora de valor.

Influencia de familiares y amigos en la creación y en el desempeño de la empresa: solo la mitad de las empresas son familiares, sin embargo, todos los empresarios valoran el apoyo moral recibido de los miembros de su familia más cercanos. Es evidente que los fundadores de las empresas familiares consideran de gran importancia que sus parientes trabajen en las mismas, pues en algunos casos contribuyen a su profesionalización, y en otros, con su cuidado al garantizar que otros empleados no familiares se abstengan de presentar actitudes oportunistas que puedan perjudicarlas; además, son estos quienes desempeñan los cargos de administración y dirección de las empresas.

Se observa en todas las empresas la carencia de elementos de gobierno corporativo; las decisiones se toman en familia o con su equipo directivo, y no existe en ellas junta directiva ni asamblea de socios. Evidentemente esta es una de las falencias más grandes que presentan las PYMES en el país, en las cuales los procesos de gobierno corporativo no son tenidos en cuenta, presentándose una gran informalidad en este sentido, y disminuyendo en gran medida sus probabilidades de dinamismo.

Influencia de la cultura regional: únicamente tres de los empresarios consideran que la cultura regional ejerce un impacto positivo en el desempeño de sus empresas. Se comprueba que los fundadores de las empresas menos dinámicas son más resistentes a la interacción con el entorno. A pesar que estudios como el de Aoyama (2009) confirman la importancia de esta variable, los empresarios entrevistados, en su mayoría, no dan mayor importancia a la misma.

Participación en redes sociales y empresariales: sólo cerca de la mitad de 
los empresarios participa en redes sociales o empresariales. Los otros empresarios temen al oportunismo que puede presentarse en los procesos asociativos y prefieren no correr riesgos. Se observa en todas las empresas una participación muy débil en la construcción de redes, situación que disminuye sus posibilidades de dinamismo, tal como lo argumentan Aidis et al (2008) en su estudio, en el que confirman la poca disposición de los empresarios a trabajar en forma colaborativa, y los efectos negativos que esta situación genera en la creación de sinergias en los mercados.

Lo expuesto anteriormente, presenta, desde la percepción de los empresarios, una mirada individual de cada una de las variables determinantes del dinamismo de sus empresas y una visión integral de todas aquellas que confluyen en el logro de dicho dinamismo.

\section{Conclusiones}

Este estudio exploratorio permitió identificar los recursos y capacidades así como las instituciones, condicionantes del dinamismo de las PYMES en Colombia. Dado su carácter de exploratorio los resultados obtenidos deben ser interpretados como una posible orientación.

Se confirma la importancia de utilizar la teoría de recursos y capacidades, y la teoría institucional como un marco teórico complementario para el análisis de dinamismo empresarial, pues como se evidencia, son diversos los factores que desde la percepción de los empresarios han impactado en el dinamismo de sus empresas. Así, que los recursos y capacidades estratégicos generan en las empresas posibilidades de crecimiento, es- pecialmente cuando están inmersos en rutinas organizativas y cuando permiten la generación de capacidades distintivas. Igualmente, se evidencia la importancia de las instituciones formales e informales en el dinamismo empresarial.

De la misma manera, este trabajo permitió corroborar la relevancia que presenta el que las PYMES logren su dinamismo lo cual será posible en la medida en que los fundadores tengan una visión amplia sobre el futuro, sean más estratégicos en sus decisiones, deseen el crecimiento empresarial, mantengan excelentes relaciones con clientes y proveedores, manifiesten una mayor disposición para acercarse a instituciones públicas y privadas, sean más conocedores del entorno y por ende de las fuerzas externas que impactan sus empresas, presenten mayor poder de negociación y cuenten con mayor legitimidad en el mercado. Igualmente es evidente la importancia de desarrollar capacidades estratégicas idiosincrásicas; creadas a partir de su propio conocimiento y experiencia, especialmente en la administración de las relaciones con sus clientes, en la transferencia de conocimiento y en el manejo de los recursos financieros.

Finalmente, el dinamismo empresarial está en función de factores internos, tales como recursos tangibles, intangibles y humanos estratégicos, y capacidades organizativas estratégicas, y de factores externos, tales como instituciones formales e informales. La diferenciación en los recursos y las capacidades organizativas estratégicas permiten la heterogeneidad de la PYME, y la generación de ventajas competitivas, cuando se conjugan con las especificidades del entorno. 
En función a lo anterior, se recomienda a los empresarios evaluar los factores internos y externos que permitan el dinamismo empresarial y, a partir de este análisis, generar estrategias para alcanzarlo. Es importante que sean conscientes de que el tamaño de sus empresas no es un condicionante para no contar con los procesos y los controles necesarios, pues como lo muestra el estudio existen claras diferencias entre las PYMES no dinámicas y las dinámicas, especialmente en su informalidad, en la falta de estructura organizativa y en la visión empresarial de sus propietarios. El sector académico debe procurar el conocimiento de la realidad de las PYMES, de sus actividades y quehaceres, para obtener un mayor impacto con sus programas de formación.

Además, es importante que dicho sector desarrolle sus programas con un enfoque eminentemente práctico, pues más que formar empresarios operativos, se requiere formar empresarios que gestionen sus empresas hacia el crecimiento, y más que cambiar sus formas de operación, se debe trabajar para cambiar en ellos actitudes y comportamientos que no favorecen el dinamismo de sus empresas. Por su parte, el sector gubernamental debe continuar con la generación de políticas sociales y económicas que las beneficien y permitan su desarrollo y crecimiento, y especialmente desarrollar mecanismos de sensibilización para que los empresarios las conozcan y se animen a utilizarlas.

Como reflexiones finales, se sugiere llevar a cabo investigaciones en otras regiones del país, con el propósito de entender su actuación desde la influencia del entorno a través del marco institucional, y estudiar el dinamismo de las nuevas empresas, ya que Colombia tiene una tasa alta de creación de empresas, y es importante identificar los factores que permiten su supervivencia y crecimiento, pues estas son las empresas del futuro.

\section{Referencias Bibliográficas}

Ahn, Mark y York, Anne (2011). Resource-based and institution-based approaches to biotechnology industry development in Malaysia. Asia Pacific Journal Management, Vol. 28, № 2, pp. 257-275.

Aidis, Ruta; Estrin, Saul y Mickiewicz, Tomasz (2008). Institutions and entrepreneurship development in Russia: a comparative perspective. Journal of Business Venturing, Vol. 23, № 6 , pp. 656-672.

Ainuddin, Azimah; Beamish, Paul; Hulland, John y Rouse, Michael (2007). Resource attributes and firm performance in international joint ventures. Journal of World Business, Vol. 42, № $1,47-60$.

Al-Mahrouq, Maher (2010). Success factors of small and medium enterprises: the case of Jordan. International Review of Economics and Business, Vol. 13, № 2, pp. 89-106.

Alvarez, Claudia y Urbano, David (2011). Environmental factors and entrepreneurial activity in Latin America. Academia Revista Latinoamericana de Administración, Vol. 48, № 1, pp. 126-139.

Alvarez, Claudia; Urbano, David; Coduras, Alicia y Ruiz-Navarro, José (2011). Environmental conditions and entrepreneurial activity: a regional comparison in Spain. Journal of Small Business and Enterprise Development, Vol. 18, № 1, pp. 120-140. 
Aoyama, Yuko (2009). Entrepreneurship and regional culture: the case of Hamamatsu and Kyoto, Japan. Regional Studies. Vol. 43, № 3, pp. 495-512.

Baldwin, John; Bian, Lin; Dupuy, Richard y Gellatly, Guy. (2000). Failure rates for new Canadian firms, new perspectives on entry and exit. Statistics Canada. Ottawa.

Carmeli, Abraham y Tishler, Ashler (2004). The relationships between intangible organizational elements and organizational performance. Strategic Management Journal, Vol. 25, № 13, pp.1257-1278.

Congreso de la República de Colombia (2000). Ley 590 de 2000; [en línea] disponible en http://www.secretariasenado.gov.co/senado/basedoc/ley_0590_2000.html (fecha de consulta: 7 de marzo de 2014).

Congreso de la República de Colombia (2004). Ley 905 de 2004; [en línea] disponible en http://www.secretariasenado.gov.co/senado/basedoc/ley_0905_2004.html (fecha de consulta: 7 de marzo de 2014).

Departamento Administrativo Nacional de Estadística de Colombia- DANE. (2005). Estadísticas. Disponible: http://www. dane.gov.co. (fecha de consulta: 1 de marzo de 2011).

Dhawan, Rageev (2001). Firm size and productivity differential: theory and evidence from a panel of US firms. Journal of Economic Behavior and Organization, Vol. 44, № 1, pp. 269-293.

European Federation of Accountants (FEE). (2004). Avoiding business failure: a guide for SMEs; [en línea] disponible en http://www.fee.be/images/publications/sme-smp/Avoiding_Business_ Failure_041 01532005191 130.pdf (fecha de consulta: 7 de marzo de 2014).

Fischer, Eileen y Reuber, Rebecca (2003). Support for rapid growth firms: a comparison of the views of founders, government policymakers, and private sector resource providers. Journal of Small Business Management, Vol. 41, № 4, pp. 346-365.

Franco, Mario y Haase, Heiko (2009). Failure factors in small and medium-sized enterprises, qualitative study from an attributional perspective. International Entrepreneurship Management Journal, Vol. 6, № 4, pp. 503-521.

Franco-Ángel, Mónica y Urbano, David (2010). El éxito de las PYMES en Colombia: un estudio de casos en el sector salud. Estudios Gerenciales, Vol. 26, № 114, pp. 77-96.

Gans, Joshua; MacDonald, Glenn y Ryall, Michael (2008). The two sides of competition and their implications for strategy. Working paper, Melbourne Business School, Melbourne, Australia; [en línea] disponible en http://works.bepress. com/cgi/viewcontent.cgi?article= 1014\&context=michael_ryall (fecha de consulta: 7 de mrzo de 2014).

Global Entrepreneurship Monitor-GEM. (2010). Informe Colombia e Informe Mundial; [en línea] disponible en http://bibliotecadigital.icesi. edu.co/biblioteca_digital/handle/ item/5417 (fecha de consulta: el 7 de marzo de 2014).

Hofstede, Geert (1994). The business of international business is culture. International Business Review, Vol. 3, № 1, pp. 1-14.

Ibielski, Dieter (1997). So What about Small Business Productivity. National Productivity Review, Vol. 17, № 1, pp. 1-4.

Kantis, Hugo (2004). Un enfoque sistémico de la creación de empresas. En H. Kantis (Eds.). Desarrollo emprendedor: América Latina y la experiencia internacional. Banco Interamericano de Desarrollo. Washington, DC.

King, Adelaine (2007). Disentangling interfirm and intrafirm causal ambiguity: a conceptual model of causal ambiguity and sustainable competitive advantage. 
Academy of Management Review, Vol. 32, № 1, pp. 156-178.

Kunc, Martin y Morecroft, John (2010). Managerial decision making and firm performance under a resource-based paradigm. Strategic Management Journal, Vol. 31, № 11, pp. 1164-1182.

Meyer, Klaus; Estrin, Saul; Bhaumik, Sumon (2009). Institutions, resources, and entry strategies in emerging economies. Strategic Management Journal, Vol. 30, № 1, pp. 61-80.

Min, Zhu; Camp, Robert C. y Garg, Rajendar (2005). Assessing the environment for small and medium enterprises success in Sichuan, China. International Journal of Commerce and Management, Vol. 15, № 3, pp. 243-254.

Mitchell, Will y Singh, Kulwant (1993). Death of the lethargic: effects of expansion into new technical subfields on performance in a firm's base business. Organizational Science, Vol. 4, № 2, pp. 152-180.

Morgan, Neil; Kaleka, Anna y Katsikeas, Constantine (2004). Antecedents of export venture performance: a theoretical model and empirical assessment. Journal of Marketing, Vol. 68, № 1 , pp. 90-108.

Nooteboom, Bart (1994). Innovation and diffusion in small firms: theory and evidence. Small Business Economics, Vol. 6, № 5, pp. 327-347.

North, Douglass C. (1990). Institutions, institutional change and economic performance. Cambridge University Press. Cambridge, UK.

North, Douglass C. (2005). Understanding the process of economic change. Princeton University Press. Princeton, NJ.

Oyedijo, Ade (2012). Competitive strategy orientations of small and medium business owners and their performance impacts: the case of paint manufacturing SMEs in south-western Nigeria.
Business and Management Review, Vol. 1, №12, pp. 10-18.

Perez-Freije, Javier y Enkel, Ellen (2007). Creative tension in the innovation process: how to support the right capabilities. European Management Journal, Vol. 25, № 1, pp. 11-24.

Piva, Mariacristina y Vivarelli, Marco (2002). The skill bias: comparative evidence and an econometric test. International Review of Applied Economics, Vol. 16, № 3, pp. 347-357.

Rodríguez, Astrid G. (2003). La realidad de la pyme colombiana: desafío para el desarrollo. Fundes Internacional. Bogotá, Colombia.

Schwab, Klaus (2011). Global Competitiveness Report; [en línea] disponible en http://www3.weforum.org/ docs/WEF_GCR_Report_201112.pdf (fecha de consulta: 7 de marzo de 2014).

Sirmon, David; Hitt, Michael y Ireland, Duane (2007), Managing firm resources in dynamic environments to create value: looking inside the black box. Academy of Management Review, Vol. 32, № 1, pp. 273-292.

St-Jean,Etienne; Pierre-André, Julien y Audet, Josée (2008), Factors associated with growth changes in "Gazelles". Journal of Enterprising Culture, Vol. 16, № 2, pp.161-188.

Suliyanto, Agus; Wulandari, Siti y Novandari, Weni (2010), Competitive strategy model for purbalingga batik. Economic Journal of Emerging Marketing, Vol. 2, № 2, pp. 169-185.

Thornton, Patricia; Ribeiro-Soriano, Domingo y Urbano, David (2011), Socio-cultural factors and entrepreneurial activity: an overview. International Small Business Journal. Vol. 29, № 2, pp.105-118.

Tödtling, Franz; Prud'Homme Van Reine, Peter y DörhöFer, Steffen (2011), Open 
Innovation and regional culture-findings from different industrial and regional settings. European Planning Studies, Vol. 19, № 11, pp. 18851907.

Welter, Friederike y Smallbone, David (2011). Institutional perspectives on entrepreneurial behaviour in challenging environments. Journal of Small Business Management, Vol. 49, № 1, pp. 107-125.
Wiklund, Johan; Patzelt, Holger y Shepherd, Dean (2009). Building an integrative model of small business growth. Small Business Economics, Vol. 32, № 4, pp. 351-374.

Zelealem, Temtime y Jaloni, Pansiri (2005). Managerial competency and organizational flexibility in small and medium enterprises in Botswana. Problems and Perspectives in Management, Vol. 1, №1, pp. 25-36. 